\title{
Análisis de oscilaciones interárea ante distintas alternativas de interconexión SIC-SING
}

\author{
Inter-area oscillation study for different SIC-SING interconnection \\ F. Salinas ${ }^{1 *} \quad$ C. Rahmann ${ }^{1} \quad$ L. Vargas ${ }^{1}$ \\ Recibido 16 de mayo de 2014, aceptado 19 de noviembre de 2015 \\ Received: May 16, 2016 Accepted: November 19, 2015
}

\begin{abstract}
RESUMEN
La interconexión del Sistema Interconectado Central (SIC) con el sistema interconectado del Norte Grande (SING), ha sido uno de los temas más comentados del año 2013 en el sector eléctrico chileno. En este contexto, dos son los proyectos que se encuentran en discusión: un enlace de corriente alterna (HVAC) en 500 $\mathrm{kV}$ impulsado por GDF SUEZ, y otro en corriente continua (HVDC) en $\pm 500 \mathrm{kV}$ planeado por la Comisión Nacional de Energía (CNE). Los proyectos presentan diferencias importantes en la tecnología y existen pocos estudios sobre los problemas que puedan aparecer durante la operación por el tipo de interconexión. En el presente trabajo se estudian oscilaciones de baja frecuencia mediante un análisis modal para las dos alternativas de interconexión mencionadas. Para esto se crea un modelo del sistema SIC-SING proyectado al 2019 en el software DigSilent. El estudio incluye el análisis de dos escenarios: uno donde el SIC envía $1.000 \mathrm{MW}$ al SING y otro donde el SING exporta $800 \mathrm{MW}$ al SIC. Los resultados obtenidos del análisis modal muestran que la interconexión mediante un enlace HVAC, lleva al surgimiento de un valor propio inestable de frecuencia de oscilación cercana a los $0,5 \mathrm{~Hz}$. El origen de este modo inestable se encuentra en la interconexión misma y está asociado a un grupo de máquinas térmicas del SING. Adicionalmente tanto para la conexión en AC como en DC, se observan modos de oscilación cercanos a $0,9 \mathrm{~Hz}$ débilmente amortiguados, firmemente relacionados con la evolución de ambos sistemas al 2019.
\end{abstract}

Palabras clave: Análisis modal, interconexión, oscilaciones interárea, HVAC, HVDC.

\section{ABSTRACT}

The interconnection of the Central Interconnected System (SIC for its initials in Spanish) with the Northern Interconnected System (SING) have been one of the trending topics in the year 2013 in the Chilean electric industry. Two are the projects that are on target for the interconnection; a high voltage alternating current (HVAC) $500 \mathrm{kV}$ connection, proposed by the French utility GDF SUEZ, and a high voltage direct current $(H V D C) \pm 500 \mathrm{kV}$ planned by the CNE, the Chilean electric regulator entity. Both projects have important differences in technology and there's not too much technical evidence of the problems that could arise in the operation, caused by the SIC-SING interconnection. Low frequency oscillations are studied in this work, using modal analysis for both interconnection projects. A SIC-SING model is developed using DigSilent software, considering 2019 as the year of a possible union of the networks. Two generation scenarios are studied; one where the central system (SIC) sends $1.000 \mathrm{MW}$ to the northern system (SING), and another one where the SING sends $800 \mathrm{MW}$ to the SIC. The modal analysis results show that interconnecting both systems with the HVAC gives arise to a $0.5 \mathrm{~Hz}$ unstable oscillation mode. This unstable mode has it origin on the interconnection and it's related to a group of steam machines on the SING system. Whether the SIC-SING system is connected with the HVAC or the HVDC, $0.9 \mathrm{~Hz}$ weakly damped modes are observed in both systems. These modes are related to the evolution of the systems at 2019.

Keywords: Modal analysis, interconnection, inter-area oscillations, HVAC, HVDC.

\footnotetext{
1 Departamento de Ingeniería Eléctrica. Universidad de Chile. Santiago, Chile. E-mail: fsalinas@ing.uchile.cl; crahmann@ing.uchile.cl; 1vargas@ing.uchile.cl

* Autor de correspondencia
} 


\section{INTRODUCCIÓN}

La interconexión SIC-SING ha sido el tema más comentado del año 2013 en el sector eléctrico nacional, principalmente debido a las discrepancias por el tipo de tecnología a utilizar, la factibilidad técnica de las alternativas así como los beneficios económicos y sociales reales del proyecto. Si bien hasta hoy existe relativo consenso sobre los aspectos positivos de una interconexión, poco se ha realizado en materia técnica para determinar si una eventual interconexión es técnicamente factible. Mas aún, el gran impulsor de este proyecto, la compañía francesa GDF SUEZ, aseguró a comienzos del año pasado que una unión en $500 \mathrm{kV}$ en alterna cumpliría con la norma técnica y no generaría problemas de estabilidad [1]. Estos resultados han generado ciertas dudas, principalmente cuando se considera la experiencia internacional existente con enlaces de larga distancia en alterna [2-5] los que, en su mayoría, concluyen que conexiones de larga distancia entre sistemas de potencia grandes tienen un impacto profundo en la dinámica del sistema. En la mayoría de los casos surgen nuevos problemas tanto en la operación normal como durante contingencias, no observados antes de la interconexión. Lo anterior podría disminuir los beneficios de una interconexión al obligar a los operadores del sistema a restringir la transferencia entre áreas o incluso a separar los sistemas luego de una falla.

Uno de los fenómenos más observados desde que se comenzaron a interconectar grandes sistemas de potencia son las oscilaciones de baja frecuencia. Dentro de los factores claves en dichas oscilaciones se encuentran la sintonización de los diferentes controladores del sistema así como la topología del mismo. Estas oscilaciones de baja frecuencia no solo aparecen después de contingencias, sino que también en condiciones normales de operación, dificultando enormemente la tarea a los operadores de red. Considerando que la interconexión SICSING tendría zonas separadas por más de 3.000 $\mathrm{km}$ de distancia con varios tramos caracterizados por líneas de interconexión débiles, unido a la gran cantidad de máquinas térmicas con pobre desempeño dinámico en el SING, resulta evidente que la operación de ambos sistemas en sincronismo podría generar problemas importantes tanto en condiciones normales de operación como durante contingencias.
En el presente trabajo se analizan nuevos modos de oscilación en el sistema interconectado SICSING al año 2019 mediante un análisis modal para dos alternativas de interconexión: una, en HVAC basada en el proyecto de GDF SUEZ; y otra, en HVDC basada en la propuesta de la Comisión Nacional de Energía (CNE). Los resultados son verificados mediante simulaciones dinámicas en el dominio del tiempo. Se analizan distintos puntos de operación dependiendo del flujo de potencia por el enlace.

El trabajo se estructura de la siguiente manera. En la Sección II se resumen los conceptos básicos para comprender el origen de las oscilaciones de baja frecuencia y su efecto a nivel sistémico. En la Sección III se estudia la teoría tras el análisis modal y el estudio de oscilaciones de baja frecuencia. En la Sección IV se presenta la metodología utilizada. Luego, en la Sección V se presentan los modelos de interconexión, la representación SIC-SING implementada en DigSilent y un resumen de los casos de estudio. En la Sección VI presentan los principales resultados obtenidos y posteriormente, en la Sección VII se realiza un análisis de sensibilidad de los resultados. Por último, en la sección VIII se presentan las principales conclusiones de este trabajo.

\section{OSCILACIONES DE BAJA FRECUENCIA [3]}

Las oscilaciones en redes eléctricas han sido observadas desde los inicios de la era eléctrica. Originalmente, las máquinas interconectadas se encontraban cerca, y las oscilaciones observadas se encontraban en rangos entre 1 y $2 \mathrm{~Hz}$. Para evitar que estas oscilaciones crecieran se incluyeron enrollados amortiguadores en los rotores de los generadores. Su función es oponerse a los cambios de velocidad actuando de acuerdo al mismo principio que el rotor de una máquina de inducción. Dichos amortiguadores absorben la energía asociada a las oscilaciones del sistema reduciendo por ende su amplitud. Con el pasar de los años, la seguridad y confiabilidad de los sistema eléctricos de potencia adquirió importancia, por lo que se comenzó a exigir que los sistemas tuviesen la capacidad de recuperarse ante fallas despejadas por medio de relés. Los reguladores rápidos de voltaje se usaban para prevenir que los generadores del sistema 
perdieran sincronismo después de una falla. Sin embargo, los sistemas de excitación rápidos tienden a reducir el amortiguamiento de las oscilaciones (principalmente entre generadores cercanos), por lo que se introdujeron los llamados estabilizadores de potencia (PSS por sus siglas en inglés), controles especialmente diseñados para amortiguar dichas oscilaciones.

Posteriormente, las compañías eléctricas descubrieron que la interconexión de distintos sistemas eléctricos de potencia (SEP) les permitía obtener beneficios económicos así como una mayor seguridad en el sistema. En algunos casos las interconexiones llevaron inmediatamente a oscilaciones de baja frecuencia con amplitud creciente, lo que impedía mantener la interconexión. En algunas ocasiones bastó con disminuir las ganancias de los reguladores de voltaje para lograr que la interconexión funcionase. Sin embargo, muchos otros casos tuvieron que esperar la factibilidad técnica de los enlaces en corriente continua para lograr una conexión segura.

Al estudiar oscilaciones, los ingenieros de la época notaron que los controladores que antes no ejercían ninguna influencia por sus tiempos de actuación lenta, como los asociados a los reguladores de velocidad de las turbinas, tenían gran importancia al realizar interconexiones de larga distancia. En particular, se observó que las turbinas a vapor afectaban oscilaciones en torno a $0,5 \mathrm{~Hz}$. Las oscilaciones de baja frecuencia en los SEP se clasifican de acuerdo a la fuente de la oscilación según [4]:

- Modos locales: asociados a oscilaciones de un generador o las unidades de una central con respecto al resto del sistema. Se encuentran usualmente en el rango entre 0,7 y $2 \mathrm{~Hz}$.

- Modos interárea: varias máquinas en una parte del sistema oscilan contra los generadores en una zona distinta del sistema. Esto ocurre comúnmente entre sistemas con interconexiones largas y débiles. El rango usual de las oscilaciones es entre 0,1 y $0,7 \mathrm{~Hz}$.

- Modos de control: asociados a controladores con una mala sintonización de parámetros o diseño del mismo.

- Modos torsionales: asociado con las componentes rotacionales del eje turbina-generador.

\section{ESTUDIO DE OSCILACIONES DE BAJA FRECUENCIA}

En general, el tipo de oscilaciones que aparecen en los sistemas de potencia dependen del tipo de perturbación a que este se ve enfrentado [7]. El análisis de las oscilaciones puede ser realizado por medio de simulaciones no lineales en el dominio del tiempo. Sin embargo, dichas simulaciones pueden consumir muchos recursos en términos de tiempo y horas hombre, especialmente cuando se consideran sistemas de potencia reales con miles de barras y cientos de generadores y puntos de carga distribuidos a lo largo de la red. Mas aún, los resultados obtenidos en las simulaciones dinámicas pueden ser difíciles de comprender. Debido a lo anterior, el estudio de las oscilaciones de baja frecuencia usualmente se realiza utilizando un modelo lineal del sistema mediante el llamado análisis modal. Este tipo de estudio permite identificar potenciales modos oscilatorios del sistema así como su origen mediante una versión linealizada del mismo. Para esto se considera un sistema modelado en variables de estado [8]:

$$
\begin{aligned}
& \dot{x}=A x+B d \\
& y=C x+D d
\end{aligned}
$$

donde $x$ es un vector de estado de dimensión $n \times 1$ (con $n$ el número total de estados), $A$ es la matriz de estado de dimensión $n \times n, B$ es la matriz de entradas de dimensión $n \times i$ (con $i$ el número de entradas del sistema), $C$ es la matriz de salida de dimensión $o \times n$ (con $o$ el número de salidas del sistema), $D$ es la matriz de realimentación de dimensión $o \times i, d$ es el vector de perturbación de entrada de dimensión $i \times 1$ e $y$ el vector de salida de dimensión $o \times 1$.

Los modos de oscilación del sistema pueden ser determinados mediante el análisis de los valores propios del sistema linealizado en torno a un punto de operación. Los $n$ valores propios del sistema $\{\lambda\}_{i=1}^{n}$ satisfacen la ecuación característica:

$$
\operatorname{det}(s l-A)=0
$$

Un valor real corresponde a un modo no oscilatorio, mientras que uno complejo corresponde a un modo oscilatorio. La parte real del valor propio entrega información sobre el amortiguamiento del modo 
y su parte imaginaria la frecuencia de oscilación. Asumiendo valores propios de la forma $\lambda_{i}=\alpha_{i}+$ $i \beta_{i}$, su coeficiente de amortiguamiento se puede calcular como:

$$
\zeta_{i}=-\frac{\alpha_{i}}{\sqrt{\alpha_{i}^{2}+\beta_{i}^{2}}}
$$

El coeficiente de amortiguamiento determina la razón de decaimiento de las amplitudes de oscilación. Si la parte real del modo es positiva, el amortiguamiento se torna negativo y, por ende, el sistema exhibirá oscilaciones que aumentan su amplitud en el tiempo. Por otro lado, un sistema con oscilaciones con amortiguamiento cercano a cero implicaría que luego de una perturbación, las oscilaciones mantendrán su amplitud durante un largo período. De un punto de vista operativo, las oscilaciones de potencia en un sistema son aceptables mientras disminuyan su amplitud en el tiempo. Si un modo aumenta en amplitud de forma sostenida, los operadores de red deben tomar acciones preventivas de forma de evitar un colapso parcial o total del sistema [7]. En los SEP modernos usualmente es aceptado 5\% como razón de amortiguamiento para un modo oscilatorio dado [3]. En caso de un valor menor a 5\%, los operadores deben tomar medidas correctivas para aumentar el amortiguamiento de las oscilaciones y preservar la seguridad del sistema.

Con el desarrollo presentado hasta el momento en un sistema de $n$ máquinas es posible conocer las potenciales frecuencias de oscilación del sistema, pero no el origen de las mismas en términos de los generadores o variables de estado involucradas. Para esto se utilizan los llamados factores de participación.

Para cada valor propio $\lambda_{i}$ el vector de $n$ columnas $\phi_{i}$ y el vector de $n$ filas $\psi_{i}$ que satisfacen:

$$
\begin{aligned}
& \mathrm{A} \phi_{i}=\lambda_{\mathrm{i}} \phi_{i} \\
& \psi_{i} A=\lambda_{i} \psi_{i}
\end{aligned}
$$

son llamados vector propio derecho e izquierdo respectivamente de la matriz $\mathrm{A}$ asociados al valor propio $\lambda_{i}$. Para tener una medida de la contribución o participación relativa de cualquier variable de estado en un modo de oscilación específico, usualmente se utilizan los factores de participación. Los factores de participación se expresan matemáticamente como la multiplicación de los vectores propios derecho e izquierdo de acuerdo con:

$$
P F_{i}=\phi_{i} \psi_{i}
$$

Los factores de participación son adimensionales. Son utilizados para identificar las variables de estado dominantes para un modo en particular. De esta manera, es posible seleccionar los generadores más adecuados para controlar un modo específico. Estos factores pueden ser útiles para ubicar estabilizadores de potencia (PSS por sus siglas en inglés). Por ejemplo, si para un modo en particular, el factor de participación de la velocidad del generador es cero, añadir amortiguamiento en el rotor de esa máquina no contribuirá a modificar el modo. Por otro lado, si el factor de participación es real positivo, añadir amortiguamiento en ese generador aumentará el amortiguamiento del modo [3]. La máquina que posea factores de participación positivos asociados a la velocidad será por tanto una buena opción para instalar un PSS.

\section{METODOLOGÍA}

A continuación se presenta la metodología utilizada para estudiar y caracterizar los modos de oscilación del sistema interconectado SIC-SING al año 2019. La Figura 1 presenta un diagrama de bloques de la metodología.

En primer lugar se recopilan los datos necesarios para construir un modelo dinámico del sistema interconectado SIC-SING en DigSilent a partir de fuentes oficiales disponibles. Los modelos de la interconexión se basan en los proyectos presentados por la CNE [4] y GDF SUEZ [1].

La determinación de los puntos de operación a analizar se realiza considerando dos hidrologías para el SIC en base a un despacho económico por lista de mérito. En base a esto se buscan aquellos puntos de operación caracterizados por grandes transferencias de potencia entre ambos sistemas, situación crítica en términos de amortiguamiento de las oscilaciones de baja frecuencia [3]. De los resultados obtenidos, se seleccionan dos condiciones de operación (N1000 y S800 en la Figura 1):

- N1000: caracterizado por un flujo de 1.000 MW desde el SIC hacia el SING 
- S800: caracterizado por un flujo de $800 \mathrm{MW}$ desde el SING hacia el SIC

A ambos puntos de operación se les realiza un análisis modal tanto para el caso del enlace HVDC como para el HVAC. El estudio se concentra en aquellos modos con frecuencias en el rango interárea, es decir, valores que pueden variar entre $0,1 \mathrm{y} 1 \mathrm{~Hz}$. Si bien se ha mencionado en la sección anterior que en los SEP es comúnmente aceptado 5\% como límite inferior para el amortiguamiento de oscilaciones de baja frecuencia, en este trabajo se considerará como estándar lo que dicta el código de red chileno, el cual estable que: el valor del factor de amortiguación de las oscilaciones electromecánicas en régimen permanente de pequeña señal, deberá ser como mínimo $10 \%$ [9]. Por lo tanto, de todas las frecuencias de oscilación presentes en el sistema se seleccionarán y analizarán aquellas que tengan un amortiguamiento menor al $10 \%$.

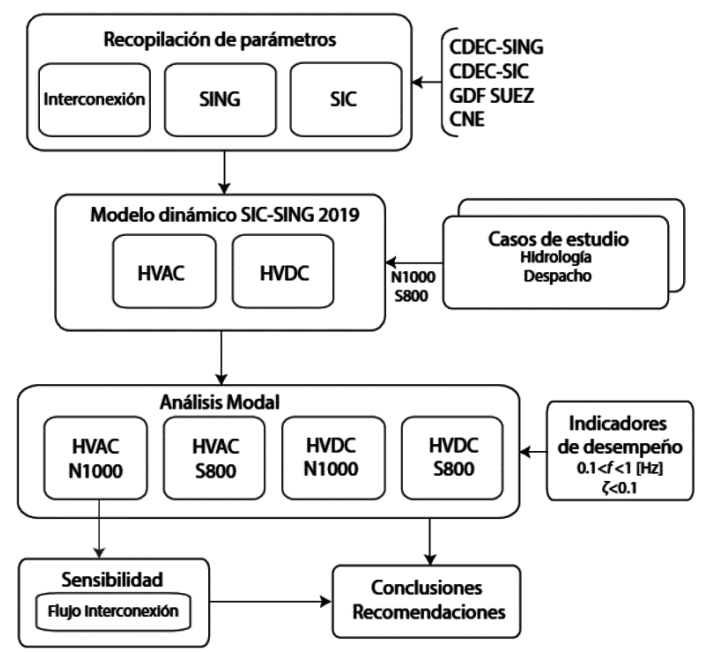

Figura 1. Metodología de estudio de oscilaciones interárea SIC-SING.

\section{CASOS DE ESTUDIO}

A continuación se presentan las características de las alternativas de conexión consideradas en el estudio.

\section{A. Enlace HVAC}

Basado en la propuesta de SUEZ [1] se considera un enlace HVAC conectado entre la S/E Cardones (SIC) y la S/E Chacaya (SING). La Figura 2 presenta un acercamiento del modelo de DigSilent de la interconexión conectando ambos sistemas.

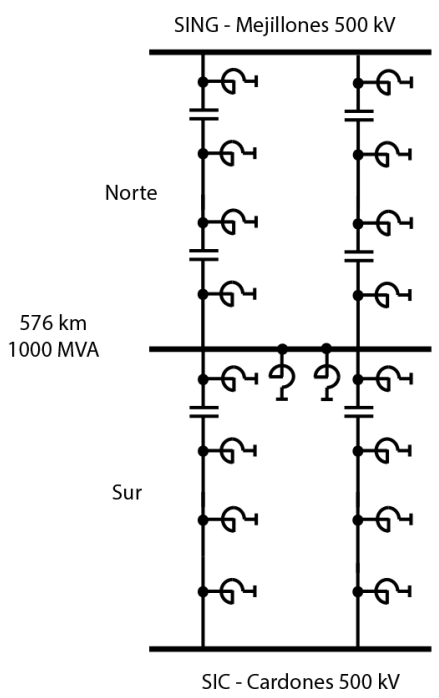

Figura 2. Enlace HVAC.

Como puede observarse en la Figura 2 la interconexión incluye una barra de compensación en la mitad del tramo debido a problemas de regulación de voltaje. El proyecto también considera compensación serie y varios reactores paralelos a lo largo de la línea. Las principales características de la interconexión se presentan en la Tabla 1.

Tabla 1. Características enlace HVAC.

\begin{tabular}{|l|c|}
\hline \multicolumn{1}{|c|}{ Elemento } & Valor \\
\hline Voltaje & $500 \mathrm{kV}$ \\
\hline Longitud & $576 \mathrm{~km}$ \\
\hline Reactores paralelo norte & $4 * 155 \mathrm{MVAr}$ \\
\hline Reactores paralelo sur & $4 * 100 \mathrm{MVAr}$ \\
\hline $\begin{array}{l}\text { Reactores S/E } \\
\text { compensadora }\end{array}$ & $2 * 110 \mathrm{MVAr}$ \\
\hline Condensadores serie norte & $2 * 55 / 2 \% \mathrm{MVAr}{ }^{2}$ \\
\hline Condensadores serie sur & $45 \% \mathrm{MVAr}$ \\
\hline Reactancia circuito & $0,4 \mathrm{Ohm} / \mathrm{km}$ \\
\hline
\end{tabular}

\section{B. Enlace HVDC}

Basado en la propuesta de la CNE [6] se considera un enlace HVDC bipolar de $\pm 500 \mathrm{kV}$ dc con cuatro subestaciones conversoras y compensación paralela del $50 \%$ de la potencia nominal, entre la S/E Encuentro (SING) y la S/E Cardones (SIC). La Figura 3 presenta un diagrama del tipo de interconexión en HVDC.

\footnotetext{
2 Porcentaje asociado a la reactancia de la línea.
} 


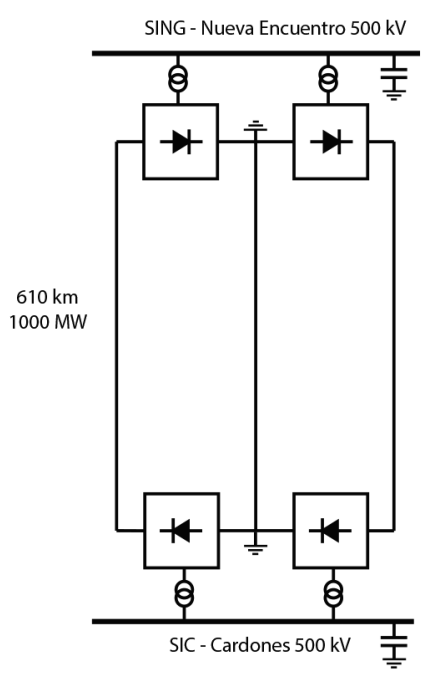

Figura 3. Enlace HVDC.

Las características de las líneas son $\mathrm{L}=0,4 \Omega / \mathrm{km}$ y $\mathrm{R}=0,019 \Omega / \mathrm{km}$.

\section{Sistema SIC-SING y casos de estudio}

El modelo resultante después de la interconexión se presenta de manera ilustrativa en la Figura 4. En la figura las obras de transmisión proyectadas a la fecha aparecen como líneas segmentadas. Se presentan las dos opciones de interconexión; un HVDC bipolar para una capacidad de 1.000 MW y una conexión HVAC de 1.000 MVA con compensación serie y paralelo. La Tabla 2 resume los casos de estudio considerados.

Tabla 2. Resumen casos de estudio.

\begin{tabular}{|l|c|c|}
\hline \multicolumn{1}{|c|}{ Caso de estudio } & \multicolumn{1}{c|}{ N1000 } & \multicolumn{1}{c|}{$\mathbf{S 8 0 0}$} \\
\hline Dirección & SIC $\rightarrow$ SING & SING $\rightarrow$ SIC \\
\hline Transferencia & $1.000 \mathrm{MW}$ & $783 \mathrm{MW}$ \\
\hline Generación hidráulica & $5.041 \mathrm{MW}$ & $3.816 \mathrm{MW}$ \\
\hline Demanda SIC & $7.293 \mathrm{MW}$ & $9.690 \mathrm{MW}$ \\
\hline Demanda SING & $2.936 \mathrm{MW}$ & $2.866 \mathrm{MW}$ \\
\hline
\end{tabular}

\section{RESULTADOS OBTENIDOS}

El modelo de ambos sistemas interconectados se implementa en el software DigSilent. Los modelos dinámicos corresponden a aquellos entregados por los respectivos CDEC [10-11]. En cuanto al modelo dinámico de las centrales de generación nuevas, se utilizan modelos existentes de centrales con características técnicas similares. Para todos los consumos del sistema se utiliza un modelo dinámico

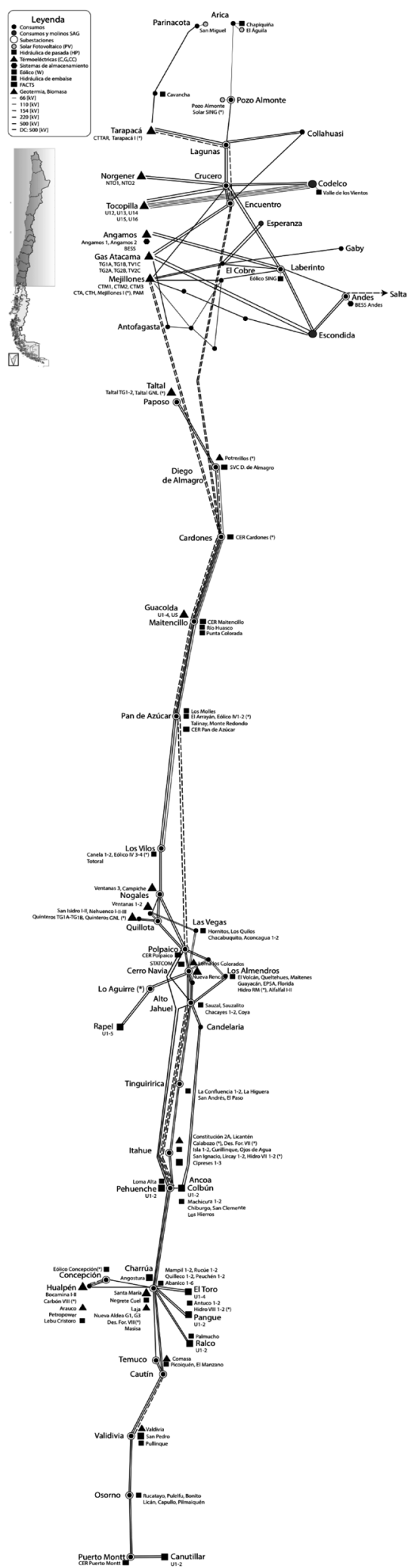

Figura 4. Sistema SIC-SING interconectado. 
de carga de potencia constante. No se incluyen modelos dinámicos detallados para generadores eólicos y fotovoltaicos.

A partir del modelo anterior se realiza un análisis modal para los casos de estudios mostrados en la Tabla 2.

\section{A. Resultados caso N1000}

A continuación se presentan los resultados de realizar un análisis modal para las dos alternativas de interconexión, considerando frecuencias menores a $1 \mathrm{~Hz}$.

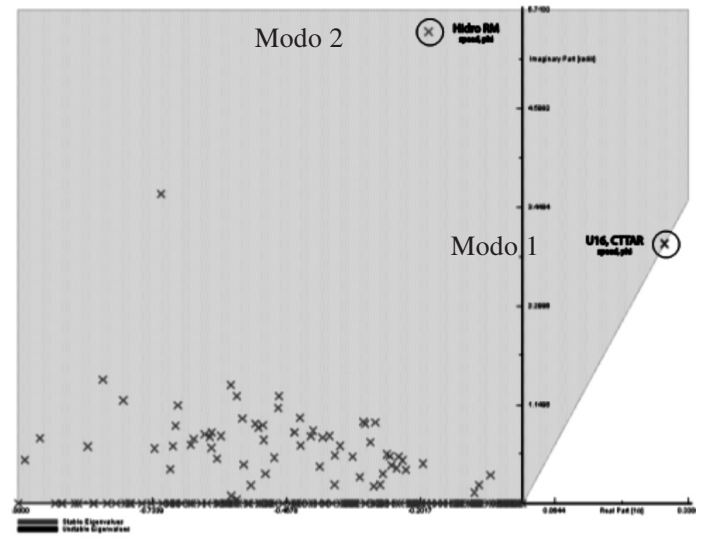

Figura 5. Valores propios del sistema, conexión en HVAC, caso N1000.

La Figura 5 presenta la parte real e imaginaria de cada valor propio del sistema para el caso de una conexión HVAC. Se restringe a los modos con frecuencias de oscilación menores a $1 \mathrm{~Hz}$. En la imagen se destacan dos modos de oscilación relevantes para el presente estudio, señalando además los generadores con participación mayor al $50 \%$ en el modo así como las variables involucradas en él mismo.

Tabla 3. Valores propios relevantes caso N1000 HVAC.

\begin{tabular}{|c|r|r|c|}
\hline Modo & & $\zeta$ & $f$ \\
\hline Modo 1 & $0,282+3,025 \mathrm{j}$ & $-9 \%$ & 0,48 \\
\hline Modo 2 & $-0,159+5,489 \mathrm{j}$ & $3 \%$ & 0,87 \\
\hline
\end{tabular}

En la Figura 5 y la Tabla 3 se destacan dos modos de oscilación: Modo 1 y Modo 2. El modo de oscilación Modo $1\left(\lambda_{1}=0,282 \pm \mathrm{j} 3,025\right)$, tiene parte real positiva lo que indica que el sistema es inestable en pequeña señal con oscilaciones de frecuencia $f=\frac{3,025}{2 \pi}=0,48 \mathrm{~Hz}$. Al ser inestable, cualquier perturbación (pequeña o grande) tendrá como resultado oscilaciones de magnitud creciente en el tiempo que podrían llevar al sistema al colapso. Los factores de participación de este modo indican que este se encuentra asociado a máquinas térmicas a vapor del SING (U16 y CTTAR, presentadas en la Tabla 7). El segundo modo de interés mostrado en la Figura 5 (Modo 2, $\lambda_{2}=-0,159 \pm \mathrm{j} 5,489$ ), tiene una frecuencia de $f=\frac{5,489}{2 \pi}=0,87 \mathrm{~Hz} \mathrm{y}$ un amortiguamiento de $\zeta=0,03$. De esta forma el Modo 2 presenta un bajo nivel de amortiguamiento que no cumpliría con el $10 \%$ mínimo requerido por la normativa chilena [9].

La Figura 6 presenta los valores propios del sistema para el caso de una conexión HVDC. La Tabla 4 resume los modos de oscilación relevantes para este caso.

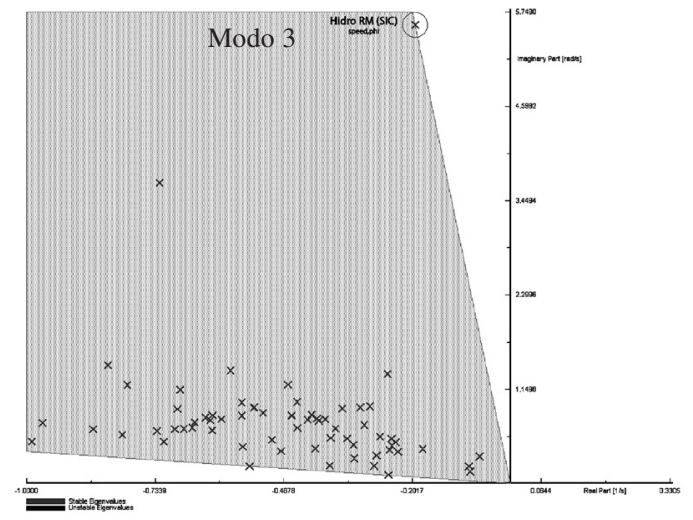

Figura 6. Valores propios del sistema, conexión en HVDC, caso N1000.

Tabla 4. Valores propios relevantes caso N1000 HVDC.

\begin{tabular}{|c|c|c|c|}
\hline Modo & $\lambda$ & $\zeta$ & $f$ \\
\hline Modo 3 & $-0,196+5,592 \mathrm{j}$ & $3 \%$ & 0,89 \\
\hline
\end{tabular}

Comparando las Figura 5 y Figura 6, se observa que al enlazar ambos sistemas con un link HVDC, el valor propio inestable (Modo 1 en Tabla 4) desaparece completamente. Se concluye por ende que dicho modo se encuentra asociado a la interconexión. Por otro lado, se observa la existencia de modos de oscilación con frecuencias de 0,87 y $0,89 \mathrm{~Hz}$ 
en ambos tipos de conexión. El origen se atribuye principalmente al aumento interno de transferencias de potencia en el SIC y a un generador nuevo que representa al proyecto Alto Maipo ("Hidro RM" en Figura 5 y Figura 6).

\section{B. Resultados caso S800}

En esta parte se realiza el mismo procedimiento utilizado para el caso N1000 pero con el escenario S800. Las siguientes figuras y tablas presentan el resultado del análisis modal para ambas alternativas de interconexión.

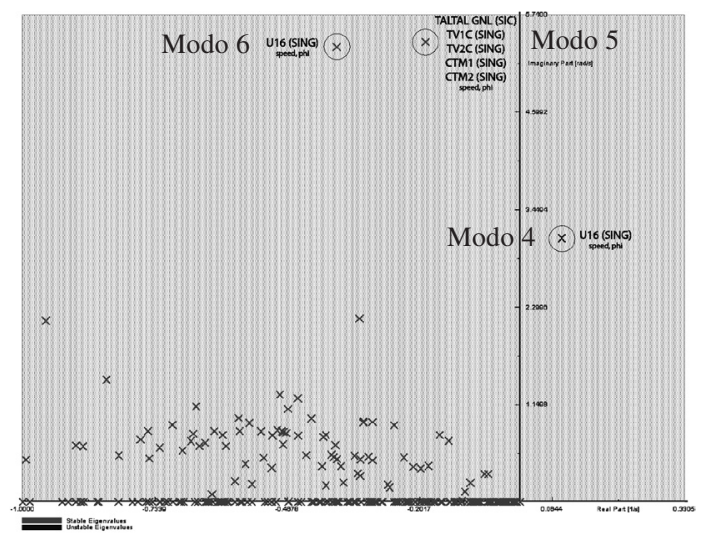

Figura 7. Valores propios del sistema, conexión en HVAC, caso S800.

Tabla 5. Valores propios relevantes caso S800 HVAC.

\begin{tabular}{|c|c|c|c|}
\hline Modo & $\lambda$ & $\zeta$ & $f$ \\
\hline Modo 4 & $0,083+3,115 \mathrm{j}$ & $-8 \%$ & 0,49 \\
\hline Modo 5 & $-0,190+5,431 \mathrm{j}$ & $3 \%$ & 0,86 \\
\hline Modo 6 & $-0,369+5,372 \mathrm{j}$ & $6 \%$ & 0,85 \\
\hline
\end{tabular}

En la Figura 7 se observa que la interconexión en HVAC genera la aparición de un modo inestable (Modo 4 en Tabla 5) con frecuencia de oscilación de $0,49 \mathrm{~Hz}$ asociado a máquinas térmicas a vapor en el SING (U16). Se visualizan a su vez dos modos de oscilación con bajo nivel de amortiguamiento con frecuencias de 0,85 y $0,86 \mathrm{~Hz}$ (ver Tabla 4). El Modo 6 aparece en el SING influenciado únicamente por la unidad de generación U16 y los generadores de la central Gas Atacama (ambos a vapor), por el alto nivel de transferencias entre estas zonas hacia

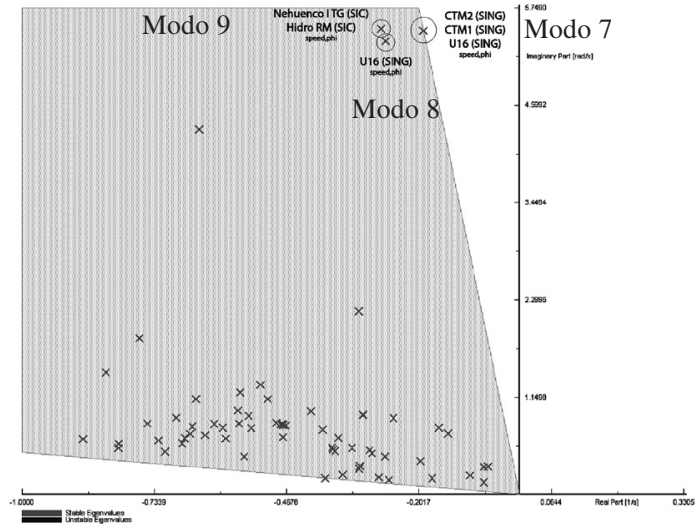

Figura 8. Valores propios del sistema, conexión en HVDC, caso S800.

Tabla 6. Valores propios relevantes caso S800 HVDC.

\begin{tabular}{|l|c|c|c|}
\hline Modo & & $\zeta$ & $f$ \\
\hline Modo 7 & $-0,193+5,476 \mathrm{j}$ & $3 \%$ & 0,872 \\
\hline Modo 8 & $-0,269+5,355 \mathrm{j}$ & $5 \%$ & 0,852 \\
\hline Modo 9 & $-0,278+5,503 \mathrm{j}$ & $5 \%$ & 0,876 \\
\hline
\end{tabular}

Mejillones (Tabla 7). Por otra parte, el Modo 5 es influenciado por una gran variedad de centrales tanto del SIC como del SING por lo que se puede asociar a un modo interárea.

El detalle de los modos observados para este caso se presenta en la Tabla 6. Al considerar la misma condición de operación con un enlace HVDC (Figura 8), se observa que el valor propio inestable existente en el caso de una conexión AC (Modo 4 en Tabla 5) desaparece completamente, indicando que es un modo cuya naturaleza está asociada a la interconexión. Se observan también modos similares a los Modos 5 y 6 del caso con HVAC, en términos de la frecuencia y el nivel de amortiguamiento. El Modo 8 es similar en características al Modo 6, relacionado a las centrales de Tocopilla (U16) y Gas Atacama (tal como el Modo 6 en el caso HVAC), mientras que los Modos 7 y 9 se relacionan con el Modo 5 del caso HVAC. Sin embargo, debido a la separación de la velocidad y ángulo entre ambos sistemas por el HVDC, el Modo 5 previamente considerado un modo interárea, se convierte en dos modos locales (Modos 7 y 9) independientes en cada sistema. 
Tabla 7. Factores de participación para modos HVAC N1000, S800 y clasificación.

\begin{tabular}{|c|c|c|}
\hline Modo & Participación & Clasificación \\
\hline Modo 1 & $\begin{array}{l}\text { SING - U16 } \\
\text { (phi } 1,00<0 \text { ) } \\
\text { SING - CTTAR } \\
\text { (phi } 0,64<30,8 \text { ) }\end{array}$ & Local SING \\
\hline Modo 2 & $\begin{array}{l}\text { SIC - Hidro RM } \\
\text { (phi } 1,00<0 \text { ) } \\
\text { SING - U16 } \\
\text { (phi } 0,55<103,6 \text { ) }\end{array}$ & $\begin{array}{l}\text { Interárea } \\
\text { SIC-SING }\end{array}$ \\
\hline Modo 4 & $\begin{array}{l}\text { SING - U16 } \\
\text { (phi } 1,00<0 \text { ) } \\
\text { SING - TV1C } \\
\text { (phi } 0,41<11,8 \text { ) } \\
\text { SING - TV2C } \\
\text { (phi } 0,41<11,8 \text { ) }\end{array}$ & Local SING \\
\hline Modo 5 & $\begin{array}{l}\text { SING - TV1C } \\
\text { (phi 0,97<173,5) } \\
\text { SING - TV2C } \\
\text { (phi 0,97<173,5) } \\
\text { SING - TG1A } \\
\text { (phi 0,42<173,6) } \\
\text { SING - TG2A } \\
\text { (phi 0,42<173,6) } \\
\text { SING - TG1B } \\
\text { (phi 0,42<173,6) } \\
\text { SING - TG2B } \\
\text { (phi 0,42<173,6) } \\
\text { SIC - Santa María } \\
\text { (phi 0,60<-56,5) } \\
\text { SING - CTM1 } \\
\text { (phi 0,77<-152,5) } \\
\text { SING - CTM3 TG } \\
\text { (phi 0,40<-147,9) } \\
\text { SING - CTM2 } \\
\text { (phi 0,75<-145,7) } \\
\text { SIC- Taltal GNL } \\
\text { (phi 0,93<-142,2) } \\
\text { SIC - Antuco 1 } \\
\text { (phi 0,38<-137,8) } \\
\text { SIC - Antuco 2 } \\
\text { (phi 0,37<-136,5) } \\
\text { SIC - Hidro VIII Región } \\
\text { (phi 0,46<-136,4) } \\
\text { SIC - Pangue 1 } \\
\text { (phi 0,31<-134,1) } \\
\text { SIC - Pangue 2 } \\
\text { (phi 0,33<-133,7) } \\
\text { SIC - Nehuenco I TG } \\
\text { (phi 0,61<-122,8) } \\
\text { (1) }\end{array}$ & $\begin{array}{l}\text { Interárea } \\
\text { SIC-SING }\end{array}$ \\
\hline Modo 6 & SING - U16 (phi 1<0) & Local U16 \\
\hline
\end{tabular}

\section{Modos interárea SIC-SING}

Dado que el HVDC separa las variables de ángulo de rotor y velocidad, no aparecen modos oscilatorios que involucren a ambos sistemas. En el caso del enlace HVAC se verifica la existencia de modos oscilatorios entre ambos sistemas. Para esto se estudian los factores de participación de forma vectorial. Se clasifica un modo interárea SIC-SING como aquel que involucra variables de estado de al menos un generador en el SIC, y otro en el SING, para una magnitud de participación mayor al 30\% y con un desfase angular mayor a 90 grados.

En la Tabla 7 se observa que los modos tienen características distintas en relación a su origen. Los modos inestables $1(0,48 \mathrm{~Hz})$ y $4(0,49 \mathrm{~Hz})$ tienen su origen en máquinas térmicas en el SING, con generadores oscilando en distintas zonas del sistema con una separación angular baja. Por esta razón se han clasificado como modos locales.

Al contrario, los Modos $2(0,87 \mathrm{~Hz})$ y $5(0,86 \mathrm{~Hz})$ corresponden a Modos interárea, involucrando a generadores de los sistemas SIC-SING con una gran separación angular en los factores de participación. Para el caso S800 (Modo 5) aumenta el número de generadores involucrados, al aumentar el número de máquinas térmicas en el sistema.

Por último, se identifica un Modo Local de la U16 para el caso S800 (Modo 6).

\section{SENSIBILIDAD}

Se estudia la relación de la potencia transferida entre el SIC y el SING para los casos de estudio. En esta parte se estudia la dependencia del modo inestable con el nivel de potencia transferida por el enlace para ambos casos de estudio (N1000 y S800) con la interconexión en HVAC.

Para determinar cómo varían los modos inestables en ambos casos de estudio (Modo 1 en N1000 y Modo 4 en S800) se disminuye progresivamente el intercambio de potencia entre los sistemas, modificando la potencia de la unidad marginal del sistema SIC-SING. A continuación se presenta el cambio en el Modo 1 (N1000) y en el Modo 4 (S800) al modificar la potencia intercambiada entre las áreas: 


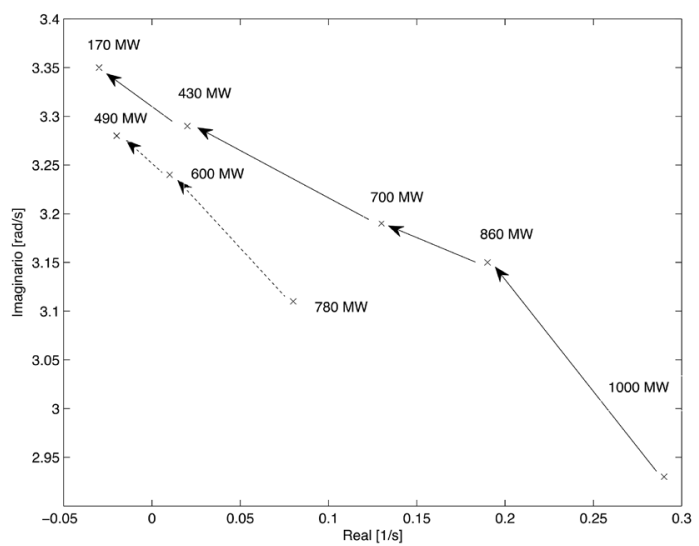

Figura 9. Sensibilidad en modos de oscilación inestables (Modo 1 y Modo 4) frente al flujo de potencia por el enlace en HVAC. Caso N1000 (línea continua) y S800 (línea segmentada).

En la Figura 9 se observa que los Modos 1 y 4 se acercan al semiplano real negativo a medida que disminuye la potencia transferida, volviéndose por ende, modos estables de oscilación. Si bien los Modos se vuelven estables, es importante destacar el bajo nivel de amortiguamiento con el que quedan razón por la que nuevamente se requeriría el uso de PSS en algunos generadores clave.

Se destaca que entre las condiciones de operación estudiadas el problema es mayor cuando el SIC envía potencia hacia el SING, volviéndose el Modo 1 estable con una transferencia en torno a los $170 \mathrm{MW}$, valor extremadamente bajo para la interconexión proyectada. Por otro lado, bajo la misma alternativa de conexión, el SING parece tener ventajas para evacuar potencia hacia el SIC. En la Figura 9 se observa que el Modo 4 se vuelve estable con transferencias en torno a los 490 MW. La diferencia entre los montos de transferencia que vuelven estables los Modos 1 y 4 se debe a la disminución de máquinas activas en el SING al recibir potencia desde el SIC, lo que genera una condición más vulnerable.

\section{CONCLUSIONES}

$\mathrm{Al}$ conectar ambos sistemas mediante un HVAC aparece un modo inestable de $0,48 \mathrm{~Hz}$ (N1000) y $0,49 \mathrm{~Hz}(\mathrm{~S} 800)$. Este modo oscilatorio tiene su origen en las máquinas de vapor del SING y el enlace HVAC, y desaparece completamente con una interconexión en HVDC. Se observó también la aparición de otros valores propios débilmente amortiguados con frecuencias de oscilación en torno a $0,9 \mathrm{~Hz}$ que no dependen del tipo de interconexión sino de la evolución de los sistemas al 2019 y de las transferencias de potencia. Del estudio se concluye que estos modos son principalmente locales en cada área ante una interconexión HVDC pero interárea para la interconexión en HVAC. Por otro lado, se observa que cuando el SING envía potencia al SIC existe un mayor número de valores propios débilmente amortiguado, por el aumento de máquinas térmicas en el sistema.

Se estudió la influencia de la potencia transferida entre el SIC y el SING en los modos inestables para el caso de un enlace HVAC. El estudio demuestra que cuando el SING envía 490 MW al SIC, el sistema pasa a ser estable en pequeña señal. Aunque la parte real del modo se vuelve negativa para estos niveles de potencia, su nivel de amortiguamiento se mantiene bajo sin cumplir niveles aceptables lo que indicaría la necesidad de activar PSS en algunos generadores. En contraste, para el caso en que el SIC envía potencia al SING, se observa que niveles de potencia bajo los 170 MW dan como resultado que el valor inestable pase a ser estable. De esta forma se concluye que el peor caso es cuando el SIC manda potencia al SING, en cuyo caso los niveles de potencia por el enlace que permitan un sistema estable en pequeña señal son muy bajos.

\section{AGRADECIMIENTOS}

Los autores agradecen el apoyo de la Comisión Nacional de Investigación Científica y Tecnológica de Chile, CONICYT (Fondap/15110019) y al Instituto de Sistemas Complejos de Ingeniería (ICM: P-05004-F, CONICYT: FBO16) durante la realización del presente trabajo.

\section{REFERENCIAS}

[1] D. Talavera. "The Mejillones-Cardones Project". GDF SUEZ. Mayo 2013.

[2] D. Thakur, K. Prasertwong and N. Mithulananthan. "Understanding low frequency oscillations in power systems". IEEE. 2013. 
[3] G. Rogers. "Power System Oscillations". Springer Science+Business Media. 2000.

[4] Siemens. "SING-SIC interconnection study for Electroandina S.A. Part A Dynamic Studies". 1999.

[5] P. Monzo and A. Giusto. "Modal analysis of the uruguayan electrical power system". Instituto de Ingeniería Eléctrica. Facultad de Ingeniería. Montevideo, Uruguay. 2010.

[6] KAS-CNE. "Análisis de necesidades de expansión del sistema de transmisión troncal SING y SIC". 2013.
[7] G. Rogers. "Demystifying Power System Oscillations". IEEE Computer Applications in Power. Vol. $9 \mathrm{~N}^{\circ}$ 3, pp. 15-30. 1996.

[8] P. Kundur. "Power System Stability and Control”. Mc-Graw Hill Inc. 1994.

[9] CNE. "Norma técnica de suministro y calidad de servicio". 2010.

[10] CDEC-SIC. "Modelo SIC DigSilent PF". 2013.

[11] CDEC-SING. "Modelo SING DigSilent PF". 2013. 